\begin{tabular}{|c|l|}
\hline Title & $\begin{array}{l}\text { Suppression of thermal denaturation of my osin and salt-induced denaturation of actin by sodium citrate in carp } \\
\text { (Cyprinus carpio) }\end{array}$ \\
\hline Author(s) & Kuwahara, Koichi; Konno, Kunihiko \\
\hline Citation & $\begin{array}{l}\text { Food Chemistry, 122(4), 997-1002 } \\
\text { https://doi.org/10.1016j.foodchem.2010.03.056 }\end{array}$ \\
\hline Issue Date & 2010-10-15 \\
\hline Doc URL & http://hdl.handle.net/2115/44026 \\
\hline Type & article(author version) \\
\hline File Information & FC122-4_997-1002.pdf \\
\hline
\end{tabular}

Instructions for use 


\title{
Suppression of thermal denaturation of myosin and salt-induced denaturation of actin by sodium citrate in carp (Cyprinus carpio)
}

\author{
Koichi Kuwahara $^{a}$ and Kunihiko Konno ${ }^{\text {b* }}$ \\ a Food Science and Technology Section, Nagasaki Prefectural Institute of Fisheries, \\ Nagasaki, Nagasaki 851-2213, Japan \\ b Laboratory of Marine Food Science, Faculty of Fisheries Sciences, Hokkaido University, \\ Hakodate, Hokkaido 041-8611, Japan \\ *Corresponding author \\ Kunihiko Konno, Tel : +81-138- 405567 ; fax: +81-138- 405567, E-mail address: \\ konnno@fish.hokudai.ac.jp
}




\section{ABSTRACT}

The effect of sodium citrate (Na-citrate) on myosin and actin denaturation in myofibrils was investigated. Na-citrate significantly suppressed the thermal inactivation of $\mathrm{Ca}^{2+}$-ATPase of carp myosin in a concentration dependent manner. The effect was greater than that of sorbitol. A similar effect was observed with myofibrils in which myosin is stabilized by F-actin binding. Na-citrate dissolved myofibrils at lower concentration than NaCl. Nevertheless, Na-citrate at $1 \mathrm{M}$ never denatured F-actin in myofibrils, while $1 \mathrm{M}$ $\mathrm{NaCl}$ denatured F-actin almost completely. Na-citrate suppressed the NaCl-induced F-actin denaturation. Sorbitol did not show such protective effect on F-actin denaturation. Moreover, Na-citrate suppressed the freeze denaturation of myofibrils at lower concentration than sorbitol. Thus, Na-citrate was proved to be superior to sorbitol. It was suggested that Na-citrate alone could substitute sorbitol as cryoprotectant in Surimi and $\mathrm{NaCl}$ as dissolving reagent of myofibril in thermal gel production.

Keywords:

Sodium citrate (Na-citrate)

Myosin

Actin

Denaturation

Sorbitol 


\section{Introduction}

Myosin is the most important component in thermal gel formation from fish and land animal meat (Samejima, Hashimoto, Yasui \& Fukazawa, 1969; Kawakami, Morita, Takahashi \& Yasui, 1971; Yasui, Ishioroshi \& Samejima, 1980; Samejima, Ishioroshi \& Yasui, 1981). It is also well accepted that myosin denaturation deteriorates the quality of the final gel products. Thus, there are many attempts to prevent myosin denaturation during the storage of meat or raw material. The satisfactory technology is surimi processing from Alaska Pollock, in which sugar or sugar alcohol was added to washed and dewatered fish meat as cryoprotectant to prevent myosin denaturation during the frozen storage (Arai, Takahashi \& Saito, 1970; Kawashima, Arai \& Saito, 1973). It is believed that addition of sugar is essential for surimi production because of unstable nature of its Alaska Pollock myosin. However, addition of sugars to surimi gives sweetness although using sorbitol instead of sucrose reduces the sweetness. It is also reported that the other compounds, such as sodium (Na-) glutamate or Na-acetate suppress myosin denaturation (Noguchi \& Matsumoto, 1970; 1971; Ooizumi, Yamamura \& Arai, 1982; Ooizumi, Nara \& Arai, 1984; Matsuura, Ooizumi \& Arai, 1984). Strong taste of Na-glutamate and weak suppressive effect of Na-acetate are their demerit. It is assumed that stabilizing effect of Na-acetate relied on carboxyl group in the structure (Ooizumi et al., 1984). Since Na-citrate is practically tasteless and its structure contains three carboxyl groups, its potential to suppress myosin denaturation is highly probable.

There is an accumulation of reports that focused on the suppression of myosin 
denaturation by additives. In contrast, there is little report on the protection of F-actin denaturation by additives. This is probably because F-actin in myofibrils is very stable compared to myosin. However, a unique F-actin denaturation upon addition of neutral salt was reported by Wakameda and Arai (1984). Treatment of actomyosin or myofibrils with $\mathrm{NaCl}$ or $\mathrm{KCl}$ at around $1.5-2 \mathrm{M}$ selectively denatures F-actin even at low temperature without accompanying myosin denaturation (Wakameda, Nozawa \& Arai, 1983). Since Na-citrate effectively dissolved squid myofibrils at lower concentrations than $\mathrm{NaCl}$ (Kuwahara, Osako, Okamoto \& Konno, 2006), Na-citrate behaved as strong salt. It is probable that $\mathrm{Na}$-citrate as salt denatures F-actin in myofibrils like $\mathrm{NaCl}$.

In order to apply Na-citrate to Surimi production and thermal gel formation, we investigated how Na-citrate addition affects thermal denaturation of myosin in the detached form or bound form to F-actin and F-actin denaturation induced by heating and salt-treatment of myofibrils

\section{Materials and methods}

\section{1. Materials}

Dorsal muscle was harvested from live carp Cyprinus carpio. All chemicals were of analytical grade and purchased from Wako Pure Chemical Industries (Osaka, Japan).

\section{2. Preparation of myofibrils and myosin}


Myofibrils were prepared from dorsal muscle of carp according to the method of Katoh, Nozaki, Komatsu and Arai (1979). Muscle mince was homogenized for 30 sec 4 times at cooling with ice-cold water on Polytron homogenizer PT 10-35 (Kinematica, Littau-Lucerne, Switzerland) in chilled buffer consisting of $0.1 \mathrm{M} \mathrm{NaCl}$ and $20 \mathrm{mM}$ Tris-HCl (pH 7.5). The homogenate was repeatedly washed by centrifugation at $3000 \mathrm{xg}$ for $5 \mathrm{~min}$ at $4{ }^{\circ} \mathrm{C}$ and suspension of the pellet. Finally, the filtrate through two layers of gauze cloth was used as myofibril preparation. Myosin was isolated from thus prepared myofibrils by the method described by Koseki, Kato \& Konno (1993). Myofibrils dissolved in $0.5 \mathrm{M} \mathrm{NaCl}, 20 \mathrm{mM}$ Tris- $\mathrm{HCl}$ (pH 7.5) was added with 5mM Mg-ATP to dissociate myosin from actin, then saturated ammonium sulfate was added to give $40 \%$ saturation to remove actin as precipitate. Myosin in the supernatant was collected by raising the saturation to $60 \%$. Myosin dissolved in and dialyzed against $0.5 \mathrm{M} \mathrm{NaCl}, 20 \mathrm{mM}$ Tris- $\mathrm{HCl}$ (pH 7.5) and the supernatant after centrifugation at $20000 \mathrm{xg}$ for $20 \mathrm{~min}$ at $4{ }^{\circ} \mathrm{C}$ was used as myosin preparation.

\section{3. Analysis of $\mathrm{Ca}^{2+}$-ATPase activity}

Myosin denaturation was assessed by $\mathrm{Ca}^{2+}$-ATPase inactivation analysis. Although Na-citrate in protein solution reduced the $\mathrm{Ca}^{2+}$-ATPase activity by chelating $\mathrm{Ca}$ ion in the reaction mixture, we confirmed that increased $\mathrm{CaCl}_{2}$ concentration to $15 \mathrm{mM}$ removed the problem. We also confirmed that inclusion of Na-citrate up to $0.1 \mathrm{M}$ in the assay medium 
unaffected the ATPase inactivation rate measurement. Accordingly, the ATPase was assayed in a medium containing, $0.5 \mathrm{M} \mathrm{NaCl}, 15 \mathrm{mM} \mathrm{CaCl}_{2}, 1 \mathrm{mM}$ ATP, $25 \mathrm{mM}$ Tris-maleate (pH 7.0), and myofibrils at about $0.1 \mathrm{mg} / \mathrm{ml}$. Thermal inactivation rate $(k)$ was calculated by assuming that the inactivation obeys the first order reaction mechanism. To analyze the suppressive effect of compounds quantitatively, thermal inactivation rates in logarithmic values were plotted against the concentrations of the compound added, and the slope of the straight line obtained was defined as E-value as Ooizumi, Hashimoto, Ogura \& Arai (1981) proposed.

\section{4. Thermal and freeze denaturation of carp myosin and myofibrils}

For thermal denaturation, myosin and myofibrils were heated at 35 and $42{ }^{\circ} \mathrm{C}$, respectively. Myofibrils were frozen stored at $-20{ }^{\circ} \mathrm{C}$ for 30 days to induce freeze denaturation. Na-citrate or other compounds were added to the above protein solution for studying their suppressive effect. As references, stabilizing effect of Na-glutamate, Na-acetate and sorbitol were also examined (Ooizumi et al., 1981). Myosin denaturation was assessed by $\mathrm{Ca}^{2+}$-ATPase inactivation analysis.

\section{5. F-actin denaturation and Salt-induced actin denaturation}

F-actin denaturation upon treatment of myofibrils with Na-citrate was studied by analyzing the $\mathrm{Ca}^{2+}$-ATPase thermal inactivation profile of the treated myofibrils upon 
subsequent heating. Carp myofibrils were mixed with 0.25 or $1.0 \mathrm{M}$ Na-citrate and incubated overnight on ice. Before the heating of $35^{\circ} \mathrm{C}$, Na-citrate concentration was diluted 3 times and $\mathrm{NaCl}$ concentration was adjusted to $0.5 \mathrm{M}$. Their inactivation profiles were assessed by $\mathrm{Ca}^{2+}$-ATPase inactivation. Salt-induced F-actin denaturation upon treatment of myofibrils with Na-citrate was studied by analyzing the $\mathrm{Ca}^{2+}$-ATPase inactivation profile of the treated myofibrils upon subsequent heating. Carp myofibrils were treated with $1.0 \mathrm{M} \mathrm{NaCl}$ together with $0,0.1,0.25$, and $0.4 \mathrm{M}$ Na-citrate in ice cold water overnight. For the analysis of F-actin denaturation upon treatment, the salt concentration of the medium was adjusted to $0.5 \mathrm{M} \mathrm{NaCl}$ and $0.25 \mathrm{M}$ Na-citrate by 3 times dilution with 20 $\mathrm{mM}$ Tris- $\mathrm{HCl}(\mathrm{pH} \mathrm{7.5)}$ at which actin denaturation by $\mathrm{NaCl}$ was negligible. Thus treated myofibrils were heated at $35{ }^{\circ} \mathrm{C}$ and their inactivation profile was compared with that of untreated myofibrils under the same conditions (Yasui, Kawakami \& Morita, 1968; Yamashita, Arai \& Nishita, 1978; Takahashi et al., 2005).

\section{6. Solubilization of muscle protein}

Carp myofibrils in $0.1 \mathrm{M} \mathrm{NaCl}, 20 \mathrm{mM}$ Tris-HCl (pH 7.5) were added by various concentrations of $\mathrm{NaCl}$ or $\mathrm{Na}$-citrate in ice cold water for $30 \mathrm{~min}$. The mixture was centrifuged at $20000 \mathrm{xg}$ for $30 \mathrm{~min}$ at $4{ }^{\circ} \mathrm{C}$. Protein content recovered in the supernatant relative to one before centrifugation was the index of solubilization (Konno, Yamanodera \& Kiuchi, 1997). To measure protein concentration of the supernatant, solubilized proteins was precipitated by centrifugation at $4000 \mathrm{rpm}$ for $30 \mathrm{~min}$ at $4{ }^{\circ} \mathrm{C}$ with $7.5 \%$ trichloroacetic 
acid, and the pellet was dissolved again with $1 \mathrm{M} \mathrm{NaOH}$. Protein concentration was estimated by using Biuret reagent (Gornall, Bardawill \& David, 1949).

To confirm the result, we repeated the same set of experiment at least three times by using different preparations, and results were presented only when the same conclusion was made by the separate experiment.

\section{Results and discussion}

\section{1. Stabilizing effect of Na-citrate on carp myosin}

First, to estimate the potential stabilizing ability of Na-citrate on myosin, its effect on myosin itself was studied. Myosin in $0.5 \mathrm{M} \mathrm{NaCl}$ was heated at $35{ }^{\circ} \mathrm{C}$ with various concentrations of Na-citrate and thermal inactivation rates of $\mathrm{Ca}^{2+}$-ATPase were estimated. Although Na-citrate in myosin solution reduced the activity by chelating $\mathrm{Ca}^{2+}$ in the reaction mixture, we confirmed that increased $\mathrm{CaCl}_{2}$ concentration to $15 \mathrm{mM}$ removed the problem. We also confirmed that inclusion of Na-citrate up to $0.1 \mathrm{M}$ in the assay medium unaffected the ATPase inactivation rate measurement. For a quantitative analysis of the suppressive effects, thermal inactivation rates in logarithmic values were plotted as a function of the concentrations used according to Ooizumi et al., (1981) (Fig. 1). The plotting for all compounds including Na-citrate gave straight lines with different slopes. The slope of the relationship for Na-citrate was steeper than those obtained with sorbitol or Na-acetate, and similar to that obtained with Na-glutamate. The results indicated that 
Na-citrate more effectively suppressed myosin denaturation than sorbitol, most popular and widely used additive. A quantitative comparison of the suppressive effects among compounds was made by using E-value. The E-value for Na-citrate as well as Na-glutamate (1.7) was greater than that for sorbitol (1.1) and Na-acetate (0.7). The E-value of Na-citrate indicated that inclusion of $1 \mathrm{M}$ Na-citrate stabilizes myosin by $10^{1.7}$ times (about 50 times), while the extent achieved by sorbitol is $10^{1.1}$ (about 13 times). Stabilizing effect of Na-citrate was found to be superior to sorbitol. As Na-glutamate with E-value of 1.7 was reported to show the largest stabilizing effect among compounds studied (Ooizumi et al., 1981), stabilizing effect of Na-citrate was comparable to it.

\section{2. Stabilizing effect of Na-citrate on myofibrils denaturation}

We further studied the suppressive effect of Na-citrate on thermal denaturation of myosin in myofibrils, namely myosin in a stabilized form through F-actin binding. The same set of experiment was conducted by using myofibrils at raised temperature of $42{ }^{\circ} \mathrm{C}$. The rates were estimated at varied concentrations of Na-citrate and their logarithmic values were plotted against the concentration (closed circles in Fig. 1). Again, the plotting gave a straight line. E-value calculated from the slope was 1.4 which was smaller than the ones obtained with myosin alone (1.7). Generally, E-values obtained with myofibrils are smaller than those obtained with myosin for any kind of compounds (Hayashi \& Konno, 2006). Probably low concentration of $\mathrm{NaCl}$ such as $0.1 \mathrm{M}$ contained in the myofibrils accelerated the inactivation slightly through reduced protection by F-actin. Practically Na-citrate 
showed a similar suppressive effect on myofibrils as on myosin alone. The result was somehow different from the case of other organic salt such as Na-glutamate or Na-acetate because their suppressive effect on myofibrils was remarkably low compared with that on myosin subfragment-1 (Hayashi \& Konno, 2006).

\section{3. Dissolving myofibrils upon addition of Na-citrate}

$\mathrm{NaCl}$ at 1.5 - $2 \mathrm{M}$ destabilizes myosin as a result of removal of the protection by F-actin (Wakameda et al., 1983; 1984). We found that Na-citrate at $1 \mathrm{M}$ did not accelerate myosin denaturation when heated as myofibrils and rather remarkably stabilized myosin in myofibrils. The fact suggested that Na-citrate did not act as ionic compound, and acted as nonionic compound. To examine the ionic properties of Na-citrate as salt, its dissolving ability of myofibrils was compared with that of $\mathrm{NaCl}$. $\mathrm{NaCl}$ at $0.25 \mathrm{M}$ dissolved myofibrillar proteins almost completely. Na-citrate also dissolved myofibrils and the concentration required was as low as $0.05 \mathrm{M}$ (Fig. 2). It was concluded that Na-citrate surely acted as ionic compound. The lower concentration of Na-citrate than $\mathrm{NaCl}$ for dissolving indicated a much higher ionic strength at the same molar concentration. This might be reasonable because citrate is a trivalent carboxylic acid giving five times greater ionic strength than $\mathrm{NaCl}$ at the same molar concentration by assuming a complete dissociation of hydrogen ion from the three carboxyl groups.

\section{4. Effect of Na-citrate on F-actin denaturaion}


Na-citrate acted as a strong salt in dissolving myofibril. Nevertheless, there was no trace of F-actin denaturation by Na-citrate addition (Fig. 1). We further examined whether the F-actin denaturation by Na-citrate occurs. The method to detect F-actin denaturation is constructed based on the following facts, 1) selective F-actin denaturation in myofibrils induces myosin detachment from F-actin, 2) myosin that lost the protection by F-actin is very unstable, and 3) inactivation rate of detached myosin and one of F-actin bound myosin is distinguished easily by the inactivation profile of $\mathrm{Ca}^{2+}$-ATPase (Murozuka \& Arai, 1976; Takahashi, Yamamoto, Kato \& Konno, 2005). Carp myofibrils were mixed with 0.25 or 1 M Na-citrate and incubated overnight on ice. F-actin denaturation in the myofibrils was judged by the existence of free myosin detached from denatured F-actin by the treatment. Existence of the detached myosin was detected by studying the $\mathrm{Ca}^{2+}$-ATPase inactivation profile of the myofibrils, namely an appearance of quick inactivation phase in the profile is the evidence of the existence of detached myosin. Their inactivation profiles are presented in Fig. 3. Irrespective of the concentrations of Na-citrate used, there was no difference in the inactivation profiles between treated and untreated myofibrils. The profile with untreated myofibrils represented the denaturation profile of F-actin bound myosin. Thus treated myofibrils with Na-citrate contained no free myosin. In other words, the salt did not denature F-actin. A slightly shallow slope for the sample treated with $1 \mathrm{M}$ Na-citrate was due to different Na-citrate concentrations in the heating medium. These are $0.33 \mathrm{M}$ (myofibrils treated with $1 \mathrm{M}$ ) and $0.083 \mathrm{M}$ (myofibrils treated with $0.25 \mathrm{M}$ ), respectively. The results clearly demonstrated that Na-citrate never caused F-actin denaturation. 


\section{5. Protection of F-actin from salt-induced denaturation by Na-citrate}

The next attempt was to test the possibility whether Na-citrate suppresses $\mathrm{NaCl}$-induced F-actin denaturation. For the purpose, myofibrils were incubated with $1 \mathrm{M} \mathrm{NaCl}$ overnight on ice together with $0,0.1,0.25$ or $0.4 \mathrm{M}$ Na-citrate. The method to detect F-actin denaturation was the same as used in Fig. 3, namely detecting free myosin in the medium as a consequence of F-actin denaturation. $\mathrm{NaCl}$ concentration for the treated myofibrils was dilution to $0.5 \mathrm{M}$ so as to stop the $\mathrm{NaCl}$-induced $\mathrm{F}$-actin denaturation. Furthermore Na-citrate concentration in the heating medium was adjusted to $0.25 \mathrm{M}$ to make the comparison of the inactivation rate easier among samples. Thermal inactivation profiles of the samples at $35{ }^{\circ} \mathrm{C}$ are shown in Fig. 4. Myofibrils without NaCl-treatment exhibited a slow inactivation representing the inactivation profile of myosin stabilized by F-actin (open circles). In contrast, myofibrils treated with $1 \mathrm{M} \mathrm{NaCl}$ exhibited a very quick inactivation phase implying the existence of very unstable free myosin in the medium (closed circles in Fig. 4). The result obtained was a confirmation that $1 \mathrm{M} \mathrm{NaCl}$ denatured F-actin selectively in myofibrils. Na-citrate at $0.1 \mathrm{M}$ practically unaffected the inactivation profile (triangles in Fig. 4). Inclusion of Na-citrate at $0.25 \mathrm{M}$ changed the profile (squares in Fig. 4). The profile showed a breaking point indicating the presence of two species of myosin with different stabilities in the heating medium. Inactivation by unstable myosin contributed mainly in an early phase, while the stable myosin determined the rate in latter phase. Unstable and stable species are myosin detached from F-actin and one bound to F-actin. The phenomenon is 
often observed when salt-induced F-actin denaturation is partial. A little slower inactivation in an early phase was due to a partial contribution of the rate of latter phase. Thus, a partial prevention of F-actin denaturation by N-citrate was proved. Amount of F-actin bound myosin could be calculated by extrapolating the line of the latter phase to heating time zero. Relative remaining activity at the point was calculated to be roughly -0.7 in logarithmic scale, which corresponds to 0.5 in ordinary scale. The calculation indicated that roughly a half of myosin in the medium was present in F-actin bound form. When 0.4 M Na-citrate was added, a quick inactivation phase disappeared from the profile and gave the same inactivation profile as untreated myofibrils (diamonds in Fig. 4). The result showed that all of myosin in the medium was in F-actin bound form, or F-actin denaturation by $\mathrm{NaCl}$ was completely suppressed by Na-citrate. Na-citrate at $0.6 \mathrm{M}$ gave the same result as $0.4 \mathrm{M}$ (data not shown). Na-citrate at $0.4 \mathrm{M}$ was proved to be high enough to prevent F-actin denaturation completely.

As the suppressive effect of Na-citrate on NaCl-induced F-actin denaturation was evident, it is reasonable to think that other compounds that suppressed myosin denaturation might suppress the F-actin denaturation similarly. We tested sorbitol. The same set of experiments as done with Na-citrate was conducted by replacing Na-citrate with sorbitol. Sorbitol concentration used was $0.82 \mathrm{M}$, which corresponds to $0.35 \mathrm{M}$ Na-citrate in stabilizing myosin denaturation (see Fig. 1). Results are presented in Fig. 5. Incubation of myofibrils with $1 \mathrm{M} \mathrm{NaCl}$ alone generated a quick inactivation phase (closed circles in Fig. 5). Inclusion of $0.82 \mathrm{M}$ sorbitol unchanged the inactivation profile showing a quick inactivation phase in the profile (triangles in Fig. 5). It was concluded that sorbitol did not 
suppress $\mathrm{NaCl}$-induced F-actin denaturation. Electrostatic interaction of Na-citrate to F-actin seemed important to stabilize actin structure. Stabilizing mechanism of Na-citrate on F-actin is uncertain at present but practically Na-citrate might be superior additive to sorbitol by its large E-value on myosin denaturation and protective effect on F-actin denaturation.

\section{6. Freeze denaturation of myofibrils and its suppression by Na-citrate}

Above results clearly demonstrated that Na-citrate strongly protects myosin both in free form and bound form to F-actin and that it protected salt-induced F-actin denaturation. Another well known factor to cause myosin denaturation is freezing. It is well established that sugars and Na-glutamate well suppress freeze denaturation of myosin (Arai et al., 1970; Kawashima et al., 1973; Ooizumi et al., 1984). Recently, we reported that ionic compounds such as sulfate ion rather accelerated the freeze denaturation of myosin in spite of the fact that the ion strongly suppressed the thermal denaturation of myosin (Torigai \& Konno, 1996; Konno, 1998; Hayashi \& Konno, 2006). It is also reported that $\mathrm{NaCl}$ in actomyosin solution promotes F-actin denaturation during the frozen storage at around -10 ${ }^{\circ} \mathrm{C}$ (Takatori, Inoue \& Shinano, 1992). As Na-citrate behaved as strong ionic compound by dissolving myofibrils very effectively, it is still possible that Na-citrate causes F-actin denaturation during the frozen storage of myofibrils, subsequently promotes myosin denaturation.

Carp myofibrils in $0.1 \mathrm{M} \mathrm{NaCl} \mathrm{pH} 7.5$ was mixed with various concentrations of 
Na-citrate and were frozen stored at $-20{ }^{\circ} \mathrm{C}$. Sorbitol, Na-glutamate and Na-acetate were also used as references (Ooizumi et al, 1984; Matsumoto, Ooizumi \& Arai, 1985). The highest concentration was $200 \mathrm{mM}$ because it is reported that freeze denaturation requires lower concentration for protection (Matsumoto et al., 1985). The remaining $\mathrm{Ca}^{2+}$-ATPase activities of the myofibrils after storage for a month were measured to assess the freeze denaturation of myosin. Activity dropped to less than $10 \%$ that of original value when stored without any additives. With increasing the concentration of Na-citrate added, the remaining activity increased. Na-citrate at $25 \mathrm{mM}$ remarkably suppressed the activity drop and $50 \mathrm{mM}$ was sufficient to keep the activity almost unchanged. The suppressive effect of sorbitol was similar to or less than that of Na-citrate. Sorbitol at $50 \mathrm{mM}$ gave the highest remaining activity. Na-glutamate also effectively suppressed the inactivation at lower concentration range showing the maximal remaining activity at $50 \mathrm{mM}$. Na-acetate weakly suppressed the inactivation requiring $150 \mathrm{mM}$ to give the maximal remaining activity. Thus, Na-citrate was proved to be an excellent compound to prevent freeze denaturation of myosin. Its effect was similar to or better than that of sorbitol (Fig. 6).

Na-citrate functions as a very strong salt in dissolving myofibrils. Nevertheless, it never caused salt-induced F-actin denaturation. Being different from inorganic ion, organic salts including Na-citrate, Na-glutamate, and Na-acetate were proved to suppress freeze denaturation of myosin as well as its thermal denaturation (Hayashi, Azuma, Koseki, \& Konno 2007).

In thermal gel formation by surimi, it is essential to dissolve myofibrillar protein prior to heating, termed as salting process. As Na-citrate well dissolved carp myofibrils like $\mathrm{NaCl}$, 
the salting with Na-citrate could be possible without using $\mathrm{NaCl}$. As Na-citrate is practically tasteless, thermal gel without salty taste can be produced by replacing $\mathrm{NaCl}$ with Na-citrate. Frozen surimi as a raw material for gel production usually contains sugar or sugar alcohol as cryoprotectant. As Na-citrate very strongly suppressed myosin denaturation in myofibrils during the frozen storage, protection of myosin denaturation by replacing sugar with Na-citrate as cryoprotectant could be possible. The use of Na-citrate as a cryoprotectant makes it possible to produce surimi without sweet taste. By using the surimi, it becomes possible to produce the thermal gel without salty or sweet taste. Such surimi will expand the application area in food industry.

Supposing that Na-citrate at $50 \mathrm{mM}$ was added to leached fish meat, spontaneous dissolving of muscle protein is expected (Fig. 2), namely surimi would be transformed to sol (dissolved surimi). Such surimi would be categorized into salt-added surimi. In Japan, $\mathrm{NaCl}$-added surimi in small quantity is produced by the special request of manufacturers. However, the surimi is not distributed widely because of its short shelf-life due to unstable nature of myosin in the surimi as a consequence of loss of protection by F-actin. As Na-citrate well protected myosin from freeze denaturation, a new type of frozen surimi, "salt-added surimi" would become practical. Study on the practical application of Na-citrate in frozen surimi production is now on going.

\section{References}

Arai, K., Takahashi, H., \& Saito, T. (1970). Studies on muscular protein of fish-III. Inhibition by sorbitol and sucrose on the denaturation of carp actomyosin during 
frozen storage. Nippon Suisan Gakkaishi, 36, 232-236.

Gornall, A. G., Bardawill, C. J., \& David, M. M. (1949). Determination of serum proteins by means of biuret reaction. Journal of Biological Chemistry, 177, 751-766.

Hayashi, K., \& Konno, K. (2006). Stabilization of myosin by ionic compounds as affected by F-actin. Fisheries Science, 72, 1306-1312.

Hayashi, K., Azuma, Y., Koseki, S. \& Konno, K. (2007). Different effect of ionic and non-ionic compounds on the freeze denaturation of myofibrils and myosin subfragment-1. Fisheries Science, 73, 178-183.

Katoh, N., Nozaki, H., Komatsu, K., \& Arai, K. (1979). A new method for evaluation of the quality of frozen surimi from Alaska pollack relationship between myofibrillar ATPase activity and kamaboko forming ability of frozen surimi. Nippon Suisan Gakkaishi, 45, 1027-1032.

Kawakami, H., Morita, J., Takahashi, K., \& Yasui, T. (1971). Thermal denaturation of myosin, heavy meromyosin and subfragment-1. Journal of Biochemistry, 70, 635-640.

Kawashima, K., Arai, K., \& Saito, T. (1973). Studies on muscular proteins of fish-X. The amount of actomyosin in frozen "Surimi" from Alaska pollack. Nippon Suisan Gakkaishi, 39, 525-532.

Konno, K,, Yamanodera, K., \& Kiuchi, H. (1997). Solubilization of fish muscle myosin by sorbitol. Journal of Food Science, 62, 980-984.

Konno, K. (1998). Suppressive effect of sulfate on fish myosin denaturation. Fisheries Science. 64, 848-849.

Koseki, H., Kato, S., \& Konno, K. (1993). Myosin extractability as a sensitive probe for the thermal denaturation of carp myofibrils. Nippon Suisan Gakkaishi, 59, 515-518.

Kuwahara, K., Osako, K., Okamoto, A., \& Konno, K. (2006). Solubilization of myofibrils and inhibition of autolysis of squid mantle muscle by sodium citrate. Journal of Food Science, 71, 358-362.

Matsumoto, I., Ooizumi, T., \& Arai, K. (1985). Protective effect of sugar on freeze-denaturation of carp myofibrillar protein. Nippon Suisan Gakkaishi, 51, 833-839.

Matsuura, M., Ooizumi, T., \& Arai, K. (1984). The protective effect of sugar against thermal denaturation of biochemical properties of carp myosin. Nippon Suisan Gakkaishi, 50, 521-529.

Murozuka, T., \& Arai, K. (1976). Purification and thermo-stability of myosin Ca-ATPase from the frozen muscle of Yellowfin tuna. Nippon Suisan Gakkaishi, 42, 65-70,

Noguchi, S., \& Matsumoto, J. J. (1970). Studies on the control of the denaturation of fish muscle proteins during the frozen storage I. Preventive effect of Na-glutamate. Nippon Suisan Gakkaishi, 36, 1078-1087.

Noguchi, S., \& Matsumoto, J. J. (1971). Studies on the control of the denaturation of fish muscle proteins during the frozen storage II. Preventive effect of amino acids and related compounds. Nippon Suisan Gakkaishi, 37, 1115-1122. 
Ooizumi, T., Hashimoto, K., Ogura, J., \& Arai, K. (1981). Quantitative aspect protective effect of sugar and sugar alcohol against denaturation of fish myofibrils. Nippon Suisan Gakkaishi, 47, 901-908.

Ooizumi, T., Yamamura, H., \& Arai, K. (1982). Cooperative protective effects of amino acid and sugar against heat denaturation of Chub mackerel myofibrils. Nippon Suisan Gakkaishi, 48, 219-226.

Ooizumi, T., Nara, Y., \& Arai, K. (1984). Protective effect of carboxylic acids, sorbitol and Na-glutamate on heat denaturation of Chub mackerel myofibrils. Nippon Suisan Gakkaishi, 50, 875-882.

Samejima, K., Hashimoto, Y., Yasui, T., \& Fukazawa, T. (1969). Heat gelling properties of myosin, actin, actomyosin, and myosin subunits in a saline model system. Journal of Food Science, 34, 242-245.

Samejima, K., Ishioroshi, M., \& Yasui, T. (1981). Relative roles of the head and tail portions of the molecule in heat-induced gelation of myosin. Journal of Food Science, 46, 1412-1418.

Takahashi, M., Yamamoto, T., Kato, S., \& Konno, K. (2005). Comparative study on thermal denaturation modes of myosin in Walleye pollack and Carp myofibrils as affected by salt concentration. Fisheries Science, 71, 662-671.

Takatori, K., Inoue, N., \& Shinano, H. (1992). Effect of KCl concentration and storage temperature on the denaturation of myosin B stored under unfrozen conditions at minus temperatures. Nippon Suisan Gakkaishi, 58, 751-758.

Torigai, M., \& Konno, K. (1996). Pyrophosphate-accelerated actin denaturation mechanism in myofibril. Fisheries Science, 62, 307-311.

Wakameda, A., Nozawa, S., \& Arai, K. (1983). Effect of neutral salts on thermal denaturation of myofibrillar Ca-ATPase of fish. Nippon Suisan Gakkaishi, 49, 237-243.

Wakameda, A., \& Aral, K. (1984). The denaturation mechanism of Carp myosin B in the presence of high concentration of salt. Nippon Suisan Gakkaishi, 50, 635-643.

Yamashita, Y., Arai, K., \& Nishita, K. (1978). Thermo-stability of synthetic actomyosins in various combinations of myosin and actin from fish, scallop, and rabbit muscles. Nippon Suisan Gakkaishi, 44, 485-489.

Yasui, T., Kawakami, H., \& Morita, F. (1968). Thermal inactivation of myosin A adenosine triphosphate in the presence of F-actin. Agricultural and Biological Chemistry, 32, 225-233.

Yasui, T., Ishioroshi, M., \& Samejima, K. (1980). Heat-induced gelation of myosin in the presence of actin. Journal of Food Biochemisty, 4, 61-78. 


\section{Figure captions:}

Fig. 1. Suppressive effect of Na-citrate on thermal denaturation of myosin and on myofibrils. Carp myosin (open symbols) and myofibrils (closed symbols and dashed line) were heated at $35{ }^{\circ} \mathrm{C}$ and $42{ }^{\circ} \mathrm{C}$, respectively in the presence of various concentrations of Na-citrate (circles), Na-glutamate (squares), Na-acetate (diamonds), and sorbitol (triangles).

Fig. 2. Solubilization of carp myofibrils with Na-citrate. Carp myofibrils (0.1 M NaCl) were incubated with additional concentrations of $\mathrm{NaCl}$ (cross marks) or Na-citrate (circles). Relative protein content in the supernatant obtained by centrifugation of the mixture to whole protein used was expressed as percent dissolved protein.

Fig. 3. Effect of Na-citrate on the F-actin denaturation in carp myofibrils. Carp myofibrils $(0.1 \mathrm{M} \mathrm{NaCl}$ ) were incubated with $0.25 \mathrm{M}$ (circles) or $1 \mathrm{M}$ (squares) Na-citrate on ice overnight. ATPase inactivation profile at $35{ }^{\circ} \mathrm{C}$ for the treated myofibrils (closed symbols) after adjusting the $\mathrm{NaCl}$ concentration to $0.5 \mathrm{M}$ was compared with those without treatment (open symbols).

Fig. 4. Suppressive effect of Na-citrate on the NaCl-induced F-actin denaturation in carp myofibrils. Carp myofibrils were incubated with $1 \mathrm{M} \mathrm{NaCl}$ together with $0 \mathrm{M}$ (circles), 0.1 $\mathrm{M}$ (triangels), 0.25 M (squares), and 0.4 M (diamonds) Na-citrate on ice overnight. ATPase inactivation profiles for thus treated myofibrils (closed symbols) after reducing $\mathrm{NaCl}$ 
concentration to $0.5 \mathrm{M}$ were analyzed. The sample without treatment was also shown (open circles with dashed line).

Fig. 5. Effect of sorbitol on the salt-induced F-actin denaturation in carp myofibrils. Carp myofibrils were treated with $1 \mathrm{M} \mathrm{NaCl}$ together with either $0 \mathrm{M}$ (circles) or $0.82 \mathrm{M}$ sorbitol (triangles). Other procedures were the same as in Fig. 4. Treated myofibrils (closed symbols) and untreated myofibrils (open symbols) were compared.

Fig. 6. Suppressive effect of Na-citrate on the freeze denaturation of carp myofibrils. Carp myofibrils (0.1 M NaCl, $20 \mathrm{mM}$ Tris- $\mathrm{HCl}(\mathrm{pH} 7.5)$ ) containing various compounds at various concentrations were frozen stored at $-20^{\circ} \mathrm{C}$ for 30 days. The compounds used were Na-citrate (closed circles), Na-glutamate (squares), sorbitol (open circles), and Na-acetate (triangles). 
Fig. 1

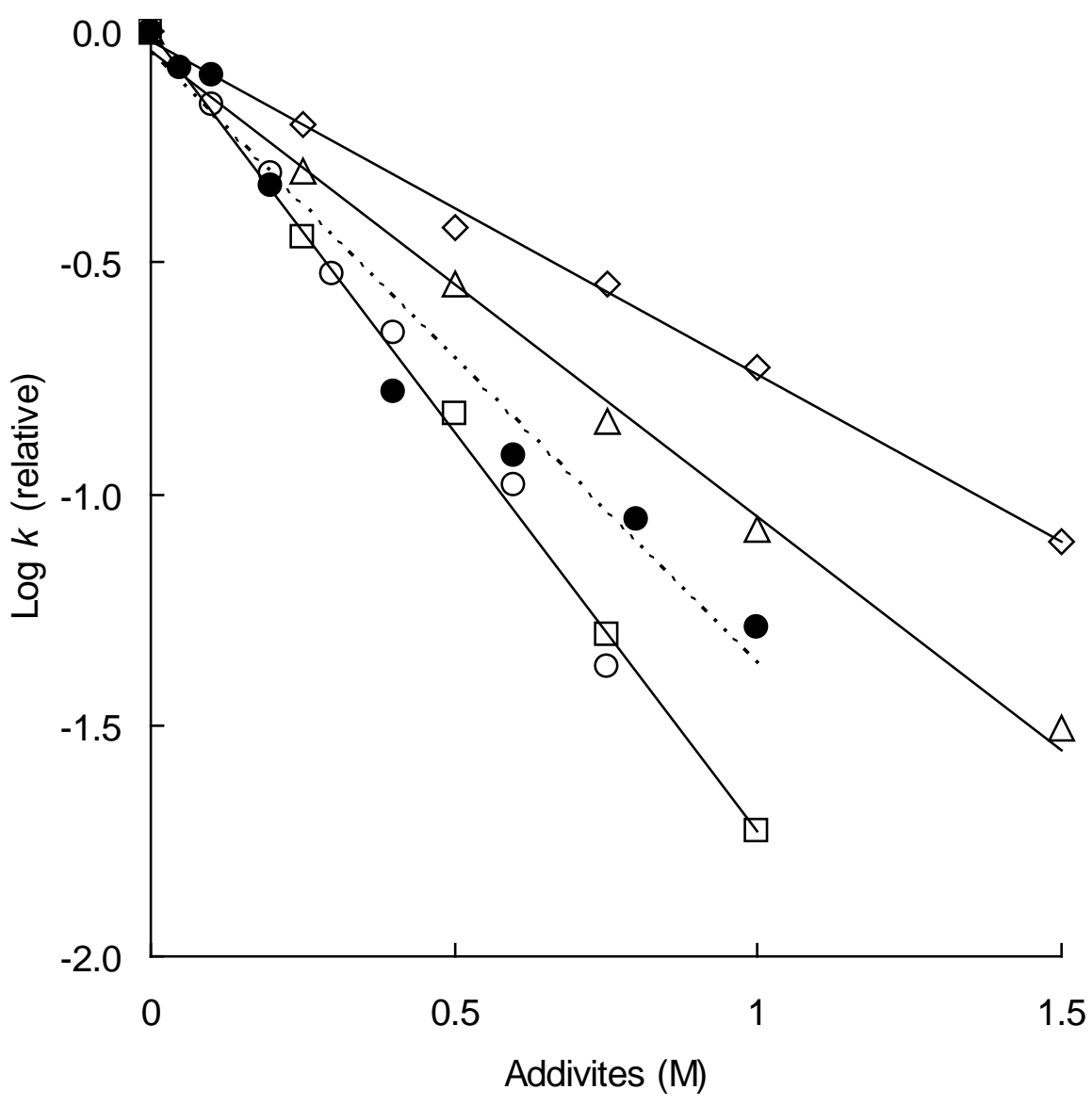


Fig. 2

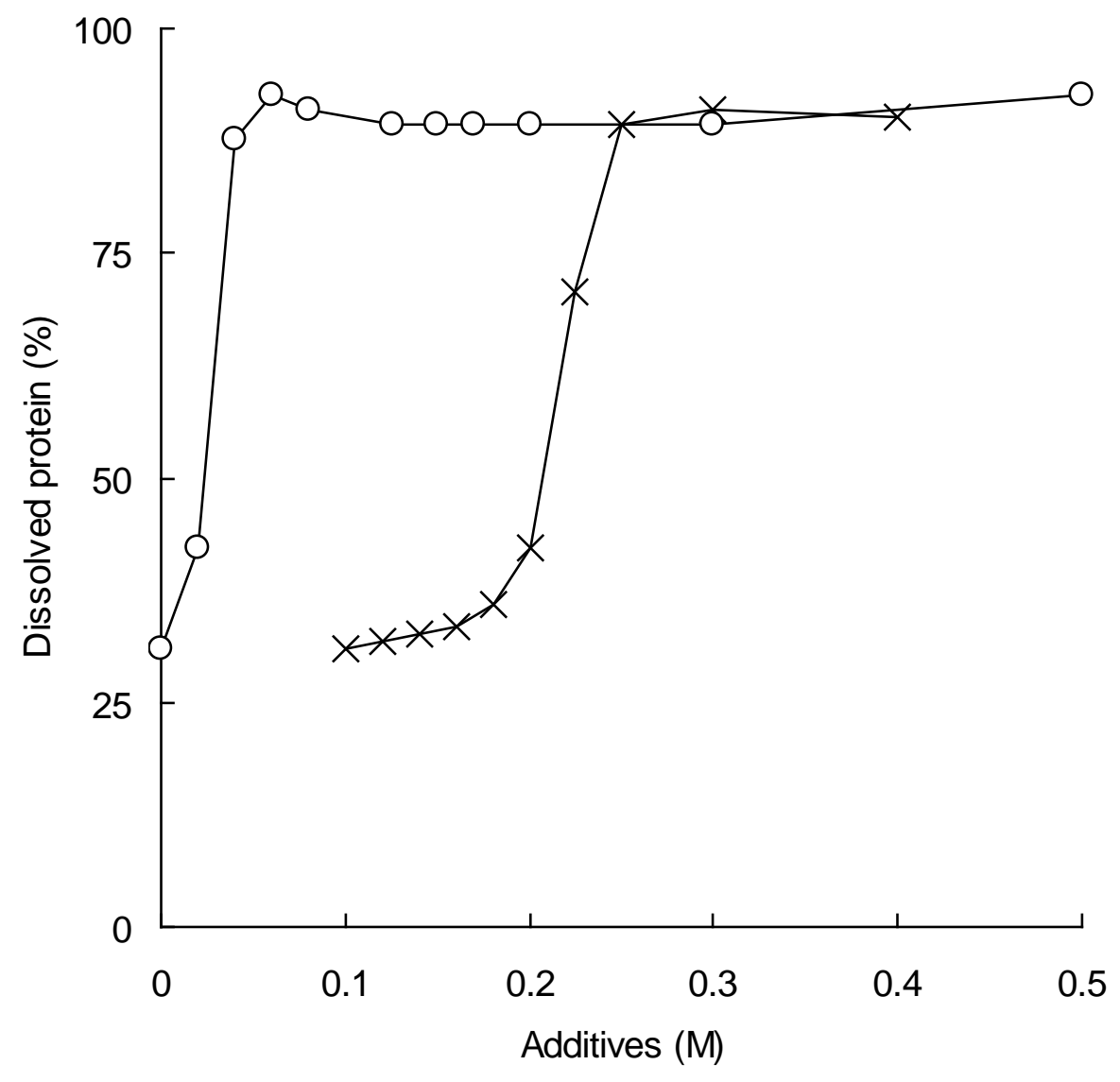


Fig. 3

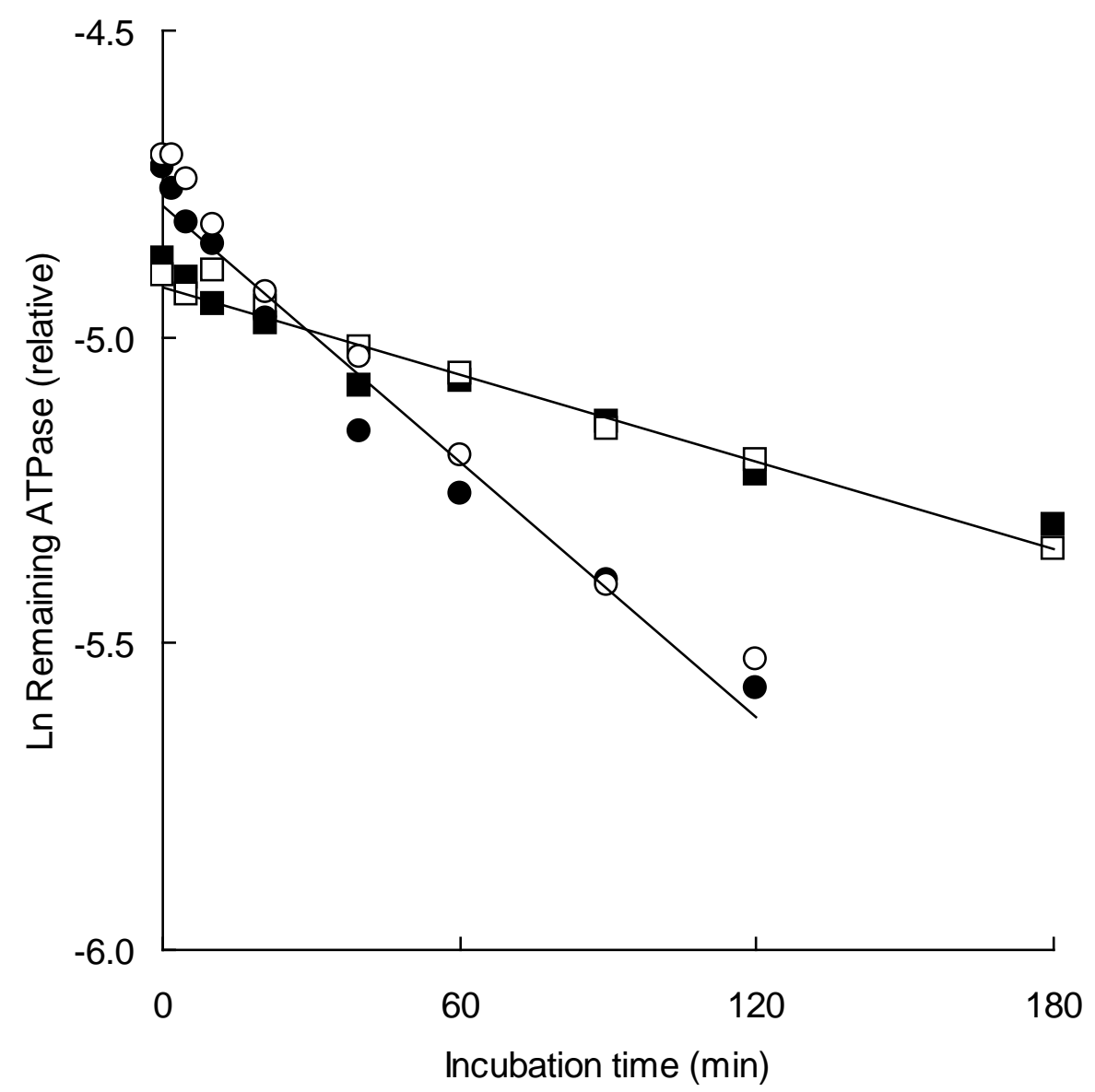


Fig. 4

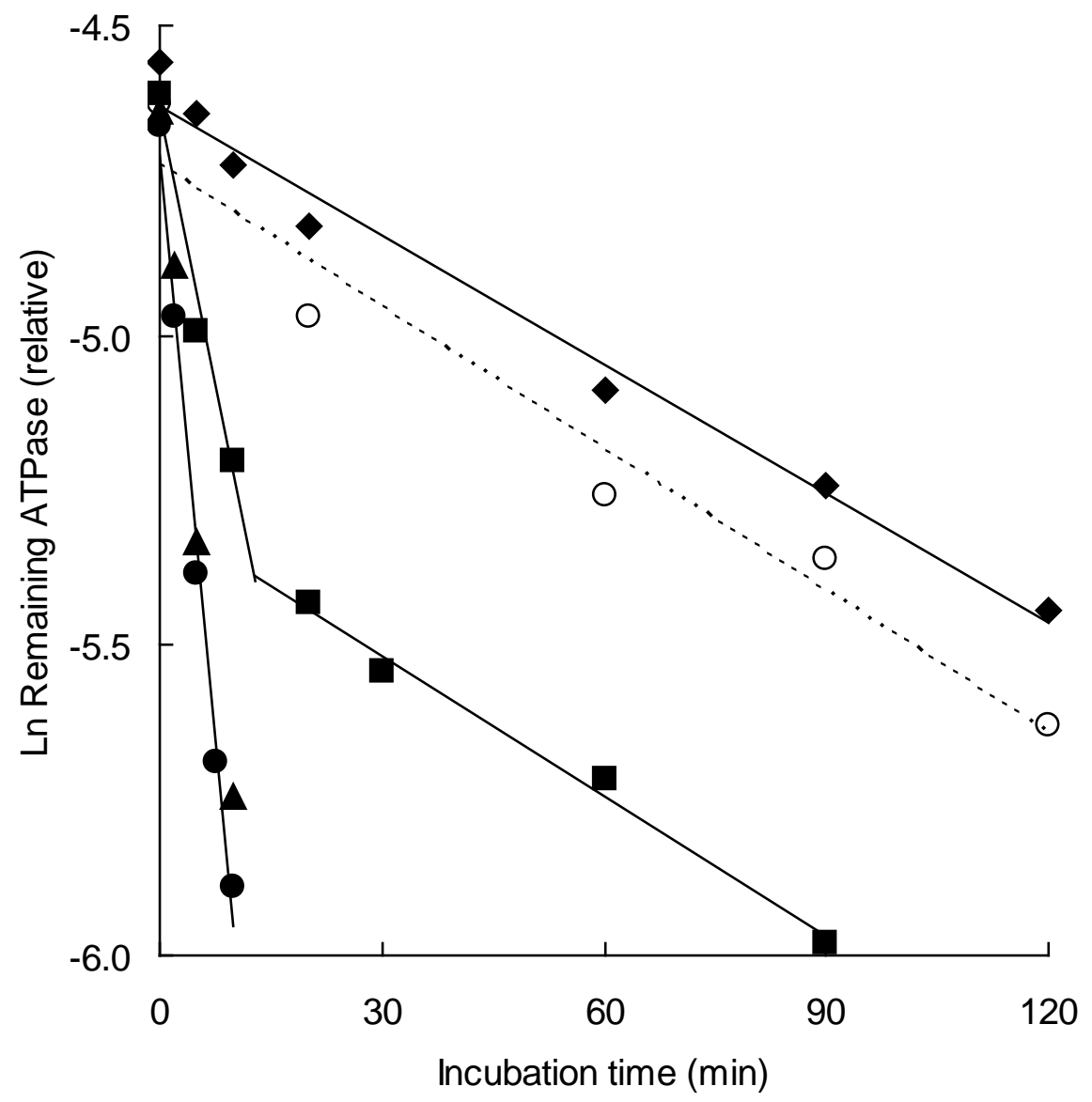


Fig. 5

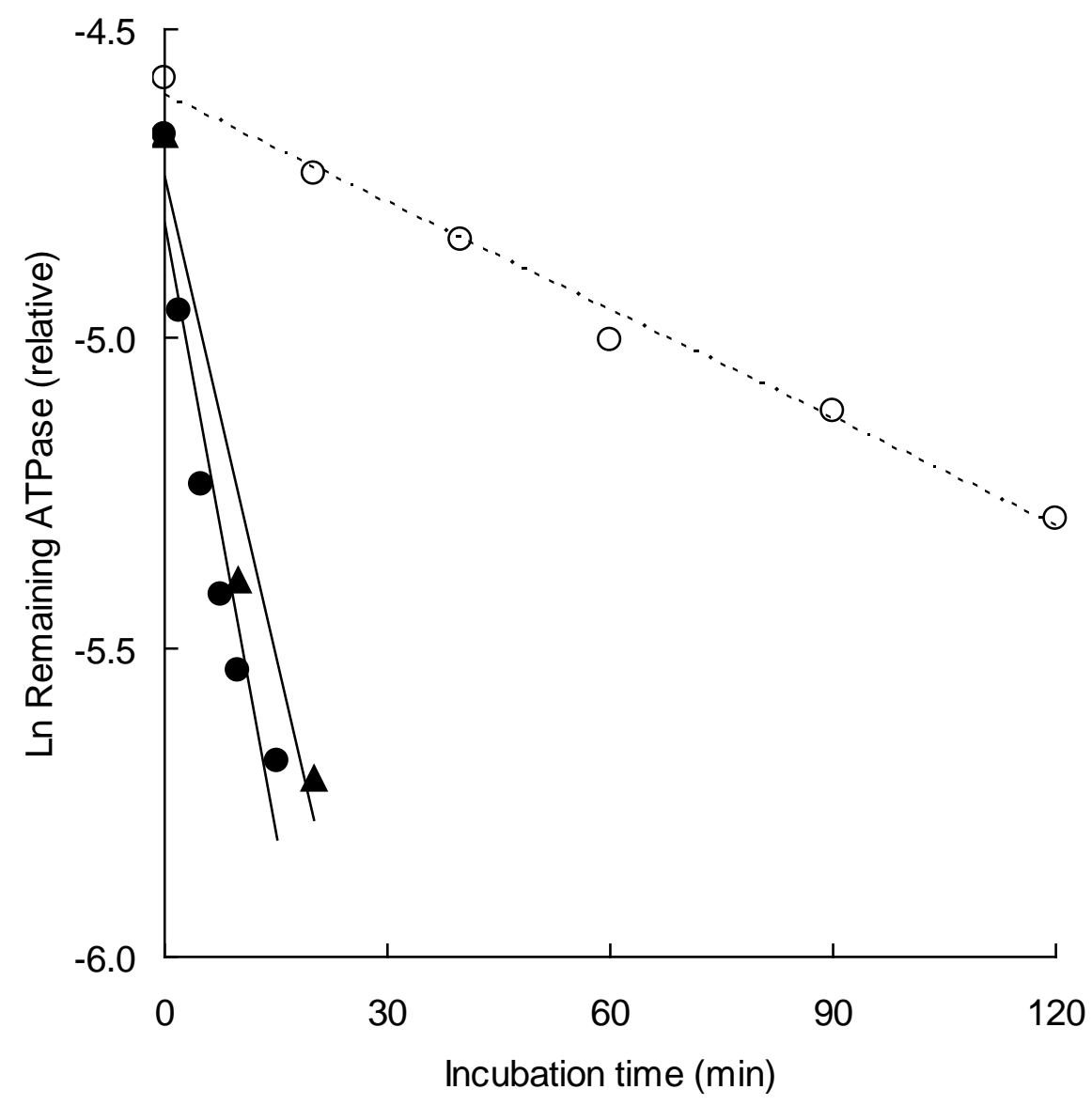


Fig. 6

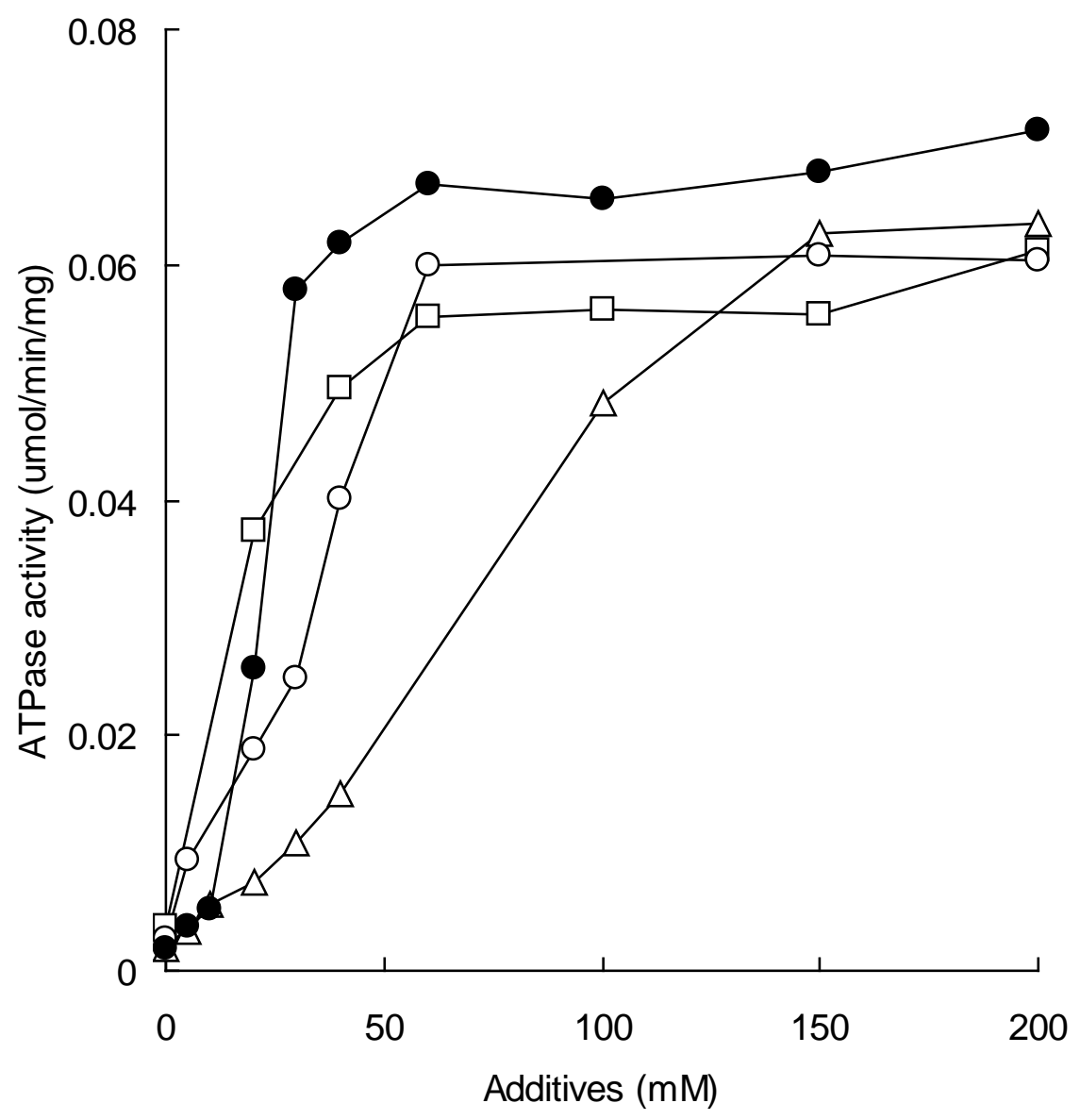

